INVESTIGACIONES

\title{
EL PROCESO DE CONSTRUCCION Y VALIDACION DE LA ESCALA DE IDENTIFICACION DE TALENTO ACADEMICO 'EDITA'
}

\section{The Process of Construction and Validation of the Academic Talent's IDENTIFICATION SCALE 'EDITA'}

\author{
O Processo de Construção e Validação da Escala de Identificação \\ do Talento Acadêmico 'Edita'
}

\section{Isabel Alegría, Siu-Lin Lay, Carlos Calderón, Manuel Cárdenas}

Universidad Católica del Norte

Comunicación con los autores: Escuela de Psicología, Facultad de Humanidades, Universidad Católica del Norte, Av. Angamos 0610, Antofagasta. E-mail: jocarde@ucn.cl

\begin{abstract}
RESUMEN
El objetivo principal de este estudio fue la construcción y validación de una escala de identificación de Talento Académico (Edita), basada en el autorreporte de las características asociadas a dicho constructo. Para ello se seleccionó una muestra compuesta por 268 estudiantes, 127 de quinto año básico y 141 de primer año medio, los que pertenecían a establecimientos educacionales municipalizados, subvencionados y particulares de la ciudad de Antofagasta. Los resultados obtenidos en el análisis de datos indican que sería una escala válida y confiable para detectar las características asociadas al Talento Académico en dicha población.
\end{abstract}

Palabras clave: Inteligencia, Talento Académico.

\begin{abstract}
The main objective of this study was the construction and validation of the Academic Talent's Identification Scale (Edita), based on the self-report of the characteristics associated with that construct. For this purpose, a sample of 268 students was chosen, 127 of fifth elementary year and 141 of the freshman year. Those students were attending public, subsidized and private schools in Antofagasta city. The results obtained through the analysis of data indicate that the scale could be considered valid and reliable to detect the characteristics associated with the Academic Talent in the above mentioned population.
\end{abstract}

Key words: Intelligence and Academic Talent.

\section{RESUMO}

O principal objetivo deste estudo foi a construção e validação de uma escala de identificação do Talento Acadêmico (Edita), baseada no auto-informe das características associadas a tal constructo. Para isso, selecionou-se uma mostra composta por 268 estudantes, 127 de quinto ano básico e 141 de primeiro ginásio, os quais pertenciam a estabelecimentos educacionais municipalizados, subsidiados e privados da cidade de Antofagasta. Os resultados obtidos na análise dos dados indicam que seria uma escala válida e confiável para detectar as características associadas ao Talento Acadêmico naquela população.

Palavras-chave: Inteligência e talento acadêmico. 


\section{INTRODUCCION}

Desde hace algunos años el sistema educacional chileno está desarrollando e implementando un proyecto de integración que permite la inclusión de niños, niñas y jóvenes con necesidades educativas especiales al aula regular. Con un enfoque educativo centrado en el aprendizaje, se incluye dentro de la educación formal a personas con discapacidad física o cognitiva como una forma de instaurar un sistema educacional orientado hacia la diversidad (Comisión de Expertos de Educación Especial, 2004). Sin embargo, dentro de estas nuevas concepciones educativas se ha dejado de lado a un grupo importante de educandos, los que se caracterizan por poseer mayores capacidades y habilidades cognitivas que el grueso de sus pares y que no ven satisfechas sus necesidades educativas con el currículo que la educación formal les ofrece.

En un afán de subsanar los vacíos educativos que deja la educación formal chilena, en algunas regiones del país se están implementando programas complementarios en apoyo a la educación formal como una forma de dar respuesta a niños, niñas y jóvenes que tienen capacidades cognitivas superiores a lo esperado para su edad, otorgándoles una educación extracurricular acorde a sus necesidades, desarrollando y estimulando sus habilidades intelectuales y socioafectivas.

Para que este servicio educativo cumpla con su objetivo es necesaria la realización de un adecuado proceso de identificación y selección de los Estudiantes que participarán en dichos programas. En Chile este proceso se caracteriza en primera instancia, por la detección de los estudiantes poseedores de Talento Académico por parte de los profesores de la institución educacional a la que el niño, niña o joven asiste en forma regular. Posteriormente a esto, se les aplica un test de inteligencia y un "Cuestionario para el Alumno" (PENTA PUC, 2004), el que a través del autorreconocimiento permite conocer las características de los postulantes, sus intereses y actitudes frente al estudio, su motivación para aprender cosas nuevas y sus expectativas. El principal problema de este último instrumento es la ausencia de estudios que den cuenta de sus propiedades psicométricas (indicadores de confiabilidad y validez).

Desde esta perspectiva, dicho proceso de selección se realiza fundamentalmente sobre la base de una medida de inteligencia (la mayor parte de las veces el Test de Matrices Progresivas de Raven), otorgándole un gran peso a las habilidades cognitivas y, por tanto, desatendiendo la medición de otras características del constructo Talento Académico que son esenciales de considerar al momento de la selección de los estudiantes. En este sentido, se hace evidente la carencia de un instrumento que logre medir e identificar tales características de manera óptima. Es por lo anterior que el objetivo principal de este trabajo es construir y validar una escala de autorreconocimiento de manifestaciones de Talento Académico que contribuya a la adecuada identificación de los niños, niñas y jóvenes talentosos.

\section{INTELIGENCIA, DOTACION Y TALENTO ACADEMICO}

Para efectos de este trabajo partimos de la base de que el concepto de talento académico debe ser adecuadamente diferenciado de los de inteligencia y dotación, siendo estos elementos una condición necesaria aunque no suficiente para poseer Talento Académico. 
A lo largo de la historia, innumerables han sido los aportes que se han hecho en relación al concepto y la forma de medir la inteligencia. Durante largo tiempo se aceptó una definición ligada a lo que se mide con los test, otorgándole gran peso a los factores genéticos y restándole importancia a los determinantes ambientales. Se trata de los denominados modelos monolíticos de inteligencia, entre los que se destaca el modelo del Factor G creado por Sperman, quien tras un análisis factorial a los test de inteligencia encontró un factor en común: la "Inteligencia General" (Sperman, 1904 citado en García \& González, 2004). Este factor G se refiere a un "conjunto de procesos comunes a todas las tareas intelectuales" y da a entender la capacidad intelectual dependiente del razonamiento abstracto, aplicación de relaciones y capacidad de percibir (Cronbach, 1998).

Estos modelos de corte más tradicional establecen una relación directamente proporcional entre inteligencia y rendimiento Académico. Como una forma de contrarrestar esto, Terman (1965) concluye que otros factores, distintos a la inteligencia, tales como la personalidad y el ambiente, determinan si un individuo alcanza o no el éxito en la vida. En esta misma línea, Weschler (1944) define inteligencia como una entidad global, siendo la capacidad del individuo para entender el mundo que lo rodea y relacionarse eficazmente con el medio. Es el producto de la genética, las experiencias socioeducacionales, las preferencias de personalidad y la motivación.

Se dio paso entonces a modelos más contemporáneos, en los cuales se establecen conceptos de inteligencia no sólo en función de habilidades cognitivas, sino que integran factores afectivos, ambientales y de personalidad (claros ejemplos son la Teoría de Inteligencias Múltiples (Gardner, 1994) y la Teoría Triádica de la Inteligencia (Sternberg, 1985).

Gardner define la inteligencia como "una habilidad, o conjunto de habilidades, que le permiten al individuo resolver problemas y proponer productos apropiados a uno o más contextos culturales" (Gardner, 1994). En su teoría, este autor plantea que no existe un solo tipo de inteligencia y que las personas poseen distintos tipos de ellas, siendo unas de mayor expresión que otras. Los tipos de inteligencia que propone son: visual, musical, corporal, interpersonal, intrapersonal, espacial, naturalista y lógico-matemática (Gardner, 1994). Desde esta perspectiva, se rescata el carácter de potencialidad que poseerían los distintos tipos de inteligencia, cobrando importancia el contexto cultural en el que se encuentre el individuo, la educación que reciba y las oportunidades de desarrollo que el medio le ofrezca.

Para Sternberg, la consideración del medio en el que el individuo se desenvuelve también es importante, ya que su concepción de inteligencia se basa en la capacidad del individuo para capitalizar las fortalezas y compensar las debilidades, cobrando importancia el uso apropiado de ellas en función de la resolución de problemas (Sternberg, 1985; Sternberg \& O’Hara, 2005). En su teoría triádica de la Inteligencia, este autor plantea tres subteorías: la de los componentes (relacionada con mecanismos mentales internos del individuo), la subteoría experiencial (la que hace mención a los niveles de experiencia o grado de conocimiento previo) y la subteoría contextual (que marca la dirección del pensamiento inteligente, adaptación, selección o modificación del ambiente). Sobre la perspectiva de estos planteamientos Sternberg y O'Hara (2005) sostienen que para que una persona sea inteligente no sólo debe tener altos niveles en una o más habilidades, sino que depende de la forma en que dichas habilidades interactúan. Por lo tanto, la inteligencia del ser humano estaría determinada por la sinergia que se produce entre habilidades analíticas, sintéticas y prácticas, las que si son bien conjugadas y contextualizadas por el individuo le permitirían una adecuada resolución de conflictos. En palabras de Sternberg 
(2000 citado en García \& González, 2004), ésta sería una inteligencia exitosa y estaría ligada al Talento Académico y se presentaría cuando en la persona existen altos niveles de sinergia entre las distintas dimensiones de su inteligencia.

La importancia de la comprensión de las teorías contemporáneas sobre inteligencia radica en la posibilidad de generar un cambio de mirada orientado hacia una perspectiva más integral de inteligencia, y por tanto de Talento Académico. Esta situación lleva a la consideración de otro tipo de variables, sin sobrestimar el valor del CI obtenido en una prueba de inteligencia.

Al hablar de Talento Académico se hace referencia a las personas con capacidades cognitivas superiores a lo normal, lo que les permite tener altos niveles de potencialidad productiva, de creatividad, autonomía y variados intereses cognitivos (Instituto Alberto Merani, 2005). Desde esta perspectiva, no sólo se está hablando de la posesión de habilidades intelectuales, sino que de una conjugación de variables que se traducen en una dotación o en un talento. Se trataría de una habilidad o desempeño excepcional en alguna dimensión general o en algún campo específico interior a esa dimensión (Bralic \& Romagnoli, 2000).

Aún nos queda aclarar la diferencia entre los conceptos de dotación y Talento, la cual se la debemos a Gagné (2000). El primero hace referencia a capacidades innatas y potenciales no entrenadas y de expresión espontánea, mientras que el segundo apunta a un desempeño concreto de carácter sobresaliente, basado en destrezas o habilidades adquiridas o desarrolladas sistemáticamente. Se estaría hablando de Talento Académico allí donde una potencialidad se traduce en una conducta talentosa superior a la de su grupo de edad, y que es producto o fruto de un entrenamiento sistemático.

Cercano a esta perspectiva encontramos los planteamientos realizados por Renzulli (2004), quien explica en su modelo de excepcionalidad "productivo-creativo" que existirían desempeños excepcionales que reflejan una interacción entre tres grupos de rasgos humanos: capacidades generales o específicas por encima del promedio, alto nivel de compromiso con la tarea y alto nivel de creatividad. De este modo, el talento académico quedaría definido por la interacción de dichos componentes.

Estas formas de concebir el constructo Talento Académico implicarían una ampliación del porcentaje de niños, niñas y jóvenes que poseerían características asociadas al Talento Académico y que se ubicarían dentro del $10 \%$ superior de su grupo de edad en dicho campo o dominio de actividad (Flanagan \& Arancibia, 2005), llegando incluso hasta un 20\% (Renzulli, 2006). En todo caso, la cifra sería muy superior al 2,5\% que tradicionalmente era considerado por otras teorías.

De este modo, el Talento Académico sería un concepto cualitativamente diferente de los de dotación e inteligencia (a los cuales subsume), y referiría a una habilidad académica superior de una persona en relación a sus pares, que se manifiesta en un campo general o en alguna dimensión específica al interior de dicho campo (Rimm, 1994; Bralic \& Romagnoli, 2000). Dicha habilidad sería de carácter adquirido o fruto de un entrenamiento (Gagné, 2000) y se caracterizaría por la interacción de dichas características excepcionales con altos niveles de creatividad y de motivación o compromiso con la tarea (Renzulli, 2004).

\section{CARACTERISTICAS DE LOS ESTUDIANTES CON TALENTO ACADEMICO}

Diversa bibliografía ha descrito las características o rasgos de los niños, niñas y jóvenes poseedores de Talento Académico, ya sean éstas del ámbito cognitivo, afectivo 
o social (George, 1992; Flanagan \& Arancibia, 2005). Estas características no se expresarían de un modo homogéneo, pudiendo observarse la presencia de una asincronía en su desarrollo (Benito, 1998; Terrassier, 1998), de modo que no todos los estudiantes talentosos manifiestan todas las características, y las que sí se manifiestan pueden hacerlo en distintos niveles de expresión (Landau, 1999).

Alonso y Benito (2004) plantean las siguientes características de los Estudiantes excepcionales: inteligencia sobre la media, diferencias cognitivas claras con el promedio tanto a nivel cualitativo como cuantitativo, mayor desarrollo en el procesamiento de la información, mayor capacidad metacognitiva a edades tempranas, insight en la resolución de problemas, buena capacidad creativa, precocidad en el desarrollo y motivación intrínseca por el aprendizaje.

A las características anteriores habría que agregar los aspectos señalados por Renzulli (2004) y que dicen referencia con la presencia de un elevado compromiso con la tarea. Se estaría hablando de motivación, la que sería constitutiva del talento y permitiría el desarrollo y la manifestación de la potencialidad, siempre y cuando sea de carácter intrínseco (Arancibia, Herrera \& Strasser, 2004). En relación a esto, se puede mencionar también la teoría propuesta por Csikszentmijalyi (1990 citado en García \& Doménech, 2002), la que hace referencia a la "experiencia óptima de flujo", que se caracterizaría por la justa adecuación entre la dificultad de la tarea y las habilidades cognitivas de quien la realiza: "La gente parece concentrarse mejor cuando las exigencias son un poco mayores de lo habitual, y son capaces de dar más de lo habitual. Si se les exige demasiado poco, la gente se aburre. Si tiene que ocuparse de demasiadas cosas, se vuelve ansiosa. El estado de flujo se produce en esa delicada zona entre el aburrimiento y la ansiedad" (Csikszentmijalyi, 1986, citado en Goleman, 1996, p. 118).

Las características que se consideran al momento de construir la Escala de Identificación de Talento Académico se dividen en tres áreas de identificación, cada una constituida por características distintivas (Centro de Estudios y Desarrollo de Talentos de la Pontificia Universidad Católica de Chile, 2004), a saber: Conocimiento y Habilidades de Aprendizaje (que contempla altos niveles de conocimientos y memoria, capacidad de atención y concentración, habilidad para trabajar con ideas abstractas, velocidad en el aprendizaje, perspicacia y capacidad de resolución de problemas), Creatividad y Productividad (que considera altos niveles de creatividad, imaginación y curiosidad, flexibilidad y fluidez en el pensamiento, pensamiento crítico y una actitud frente al riesgo desafiante) y Características Socioafectivas (representadas por altos niveles de motivación, energía, autonomía, responsabilidad y compromiso. Además, aquí se encuentra un agudo razonamiento moral y sentido de justicia, intensidad y sensibilidad emocional y el sentirse diferente ante el grupo de pares).

Basándonos en los resultados de otros estudios y en la literatura disponible sobre Talento Académico, nos proponemos contrastar ciertas hipótesis que nos sirvan como indicadores de validez para la escala elaborada. A saber: (1) Se espera encontrar una correlación positiva en el análisis de validez convergente entre la escala Edita y el Cuestionario del Alumno (PENTA UC); (2) Se espera encontrar diferencias significativas entre los estudiantes que se denominarán "Talentosos Académicamente" y la "Población General" en cuanto a la percepción de logros académicos en el área Científica-Humanista de su interés; (3) Se espera encontrar diferencias significativas entre los estudiantes "Talentosos Académicamente" y "la Población General" en relación a la percepción del 
reconocimiento social de las habilidades que poseen y la cualidad creativa de sus trabajos por sobre la de sus pares; (4) Existirán diferencias significativas entre los dos grupos antes mencionados en relación a la expresión verbal de sus emociones; (5) Se encontrarán diferencias significativas entre estudiantes "Talentosos Académicamente" y la "Población General" en cuanto al nivel de implicación familiar en su proceso educativo, y (6) No se espera encontrar diferencias significativas entre los participantes de establecimientos educacionales particulares, subvencionados y municipalizados en la escala, así como tampoco entre hombres y mujeres.

\section{METODO}

\section{MUESTRA}

La muestra quedó constituida por 268 participantes, 130 mujeres $(48,5 \%)$ y 138 hombres $(51,5 \%)$ que se encuentran cursando $5^{\circ}$ año de enseñanza básica y $1^{\circ}$ año de enseñanza media en establecimientos educacionales municipalizados $(32,8 \%)$, particulares subvencionados $(36,2 \%)$ y particulares $(31 \%)$ de la ciudad de Antofagasta. Esta muestra se divide en dos grupos, el primero de ellos constituido por 127 Estudiantes de $5^{\circ}$ año de enseñanza básica, 74 mujeres $(58,3 \%)$ y 53 hombres $(41,7 \%)$, cuyas edades fluctuaban entre 9 y 14 años (con una media de edad de 10,32 y una desviación típica de 0,65). El segundo grupo está constituido por 141 Estudiantes de $1^{\circ}$ año de enseñanza media, 56 mujeres $(39,7 \%)$ y 85 hombres $(60,3 \%)$, cuyas edades fluctuaban entre 14 y 17 años (con una media de edad de 14,47 y una desviación típica de 0,66).

\section{INSTRUMENTO}

El instrumento quedó compuesto por cuatro bloques. En el primero se recabó información relacionada con los antecedentes sociodemográficos de los participantes y sus características generales (sexo, edad, curso, establecimiento educacional, nivel socioeconómico y asignatura de mayor interés).

El segundo bloque contiene la escala Edita que tiene como característica fundamental el autorreconocimiento por parte de los participantes de las manifestaciones intelectuales y socioafectivas que tienen las personas poseedoras de Talento Académico. En su versión original quedó constituida por 76 ítems que toman forma de una escala Likert de seis puntos, con opciones de respuesta que van desde el 1 ("totalmente de acuerdo") hasta el 6 ("totalmente en desacuerdo"). Estos ítems están distribuidos en las siguientes dimensiones teóricas: Conocimiento y Habilidades de Aprendizaje (27 ítems), Creatividad y Productividad (23 ítems) y Características Socioafectivas (26 ítems).

El tercer bloque quedó constituido por cinco preguntas que se utilizaron como indicadores de validez. Estas preguntas recogen información sobre: (1) el grado de implicación familiar en su proceso educativo; (2) la obtención de logros académicos representada por su promedio de notas y por la percepción de dichos logros; (3) el grado de expresión de sus emociones, (4) la percepción de otros sobre la creatividad de sus trabajos y (5) el reconocimiento social de sus habilidades. Estas preguntas fueron planteadas con alternativas de respuesta que van desde el 1 ("Totalmente de acuerdo") hasta el 6 ("Totalmente en desacuerdo"). 
El cuarto bloque quedó constituido por "Cuestionario para el Alumno" y tiene como finalidad permitirnos realizar análisis de validez convergente, ya que este instrumento es utilizado actualmente en Chile para la selección de estudiantes con Talento Académico en programas destinados a ellos. Esta escala, constituida por 45 reactivos, a su vez está conformada por cuatro secciones. La primera de ellas con 25 ítems destinados al autorreconocimiento de las características de Talento Académico. La segunda compuesta por 11 ítems que indagan intereses y actitudes frente al estudio de los sujetos. Ambas secciones se presentan en formato Likert de cuatro alternativas de respuestas, las que fluctúan desde el 1 ("muy de acuerdo") hasta el 4 ("muy en desacuerdo"). La tercera sección con 8 ítems destinados a la descripción de la motivación para aprender cosas nuevas en los participantes, presentadas en una escala Likert de cuatro puntos que oscilan entre el 1 (“definitivamente sî") y el 4 (“definitivamente no"). La cuarta sección está conformada por un ítem que indaga en las expectativas de estudios académicos que tienen los sujetos, mediante la selección de una de las cinco alternativas que se ofrecen, que van desde estudiar hasta $2^{\circ}$ año de enseñanza media hasta seguir estudiando una vez terminada la universidad.

\section{PROCEDIMIENTO}

La prueba fue presentada a los estudiantes como parte de un estudio que tenía como objetivo pesquisar necesidades educativas especiales en estudiantes de la educación formal. La aplicación de carácter colectivo se realizó en el espacio de aula del que cada curso dispuso en su respectivo establecimiento educacional y en horario de clases.

\section{RESULTADOS}

\section{FIABILIDAD}

El coeficiente de confiabilidad alfa de Cronbach obtenido para la escala Edita fue de ,93. Del total inicial de ítems se eliminaron todos aquellos con correlación ítem-total inferior a ,25, quedando la escala final integrada por 61 de los reactivos iniciales. La correlación ítem total osciló dentro de un rango de ,26 y ,61.

Se realizaron además análisis de consistencia para cada una de las tres subescalas que componen esta prueba. Los coeficientes Alfa de Cronbach fueron de: ,87 para la dimensión Conocimiento y Habilidades del aprendizaje, de ,84 para la dimensión Creatividad y productividad, y de ,75 para la subescala Socioafectiva.

\section{ANALISIS DE CORRELACIONES}

Los análisis realizados sobre las dimensiones de la escala (ver tabla 1) indican que existe una correlación de ,7 entre las subescalas de Conocimiento y Habilidad de Aprendizaje y Creatividad y Productividad. Entre Conocimiento y Habilidad de Aprendizaje y Características Socioafectivas la correlación fue de ,62. Finalmente, la correlación existente entre las dimensiones Creatividad y Productividad y Características Socioafectivas fue de ,69. 
Tabla 1

Correlaciones entre las subescalas

\begin{tabular}{|l|c|c|c|}
\hline & Dimensión 1 & Dimensión 2 & Dimensión 3 \\
\hline Dimensión 1 & 1 &, $709(* *)$ &, $628(* *)$ \\
\hline Dimensión 2 & & 1 &, $698(* *)$ \\
\hline Dimensión 3 & & & 1 \\
\hline
\end{tabular}

** La correlación es significativa al nivel 0,01 (bilateral).

Todas estas correlaciones son significativas $(\mathrm{p}<0,001)$, lo que podría indicar que las tres dimensiones están midiendo el constructo Talento Académico y que al ser valores de correlación moderados están orientados a distintos aspectos de dicho constructo.

\section{ANALISIS FACTORIAL}

Los resultados obtenidos por medio del análisis factorial (procedimiento de extracción de componentes principales con rotación VARIMAX) revelan una estructura constituida por tres factores principales que explican el 30,18\% de la varianza. Los índices de adecuación muestral señalan un buen ajuste de los datos (test de Esfericidad de Barttlet: $\chi^{2}=4828,503 ; \mathrm{p}=, 000$ y $\left.\mathrm{KMO}=, 806\right)$.

Los resultados obtenidos por este análisis muestran una estructura factorial acorde con las dimensiones teóricas propuestas para el constructo Talento Académico (ver tabla 2). De este modo, el Factor 1 explicaría el 12,77\% de la varianza saturando en él los ítems 3, 10, 12, 13, 14, 24, 25, 35, 40, 42, 46, 58, 60, 62, 66, 69, 70, 71, 72, 73 у 75 (ver matriz de componentes en el anexo). Este factor es homologable a la dimensión Conocimiento y Habilidad de Aprendizaje y hace alusión a los niveles de conocimiento y capacidad de memoria, capacidad de atención y concentración, velocidad en el aprendizaje, habilidades para trabajar con ideas abstractas, entre otras. Pese a que el ítem 30 presenta un peso factorial elevado en el componente 3, se decidió dejarlo en la dimensión 1, ya que hace alusión a la capacidad de atención y concentración, característica relevante en el contenido teórico de esta dimensión. Situación similar es la del ítem 44 que presenta un peso factorial en el componente 2 , sin embargo dicho reactivo hace referencia a la velocidad en el aprendizaje, característica que se considera corresponde mejor a esta dimensión teórica.

El Factor 2 aludiría a la dimensión Creatividad y Productividad, haciendo referencia a imaginación, flexibilidad y fluidez del pensamiento, creatividad, actitud frente al riesgo, entre otras. Este factor explica el 8,85\% de la varianza, saturando en él los ítems 4,11 , $15,17,26,27,31,43,45,49,56,61,63,65,68$ y 76 (ver matriz de componentes en el anexo). Al igual que en la dimensión anterior los ítems 19, 47 y 74 presentan también un peso factorial elevado en el componente 1 , sin embargo se decidió dejarlos en la dimensión 2, ya que hacen alusión a la Creatividad (19 y 47) y a la actitud frente al riesgo (74), ambas características fundamentales de esta dimensión, así como también los ítems 5, 34 y 50 presentan un mayor peso factorial en el componente 3 , sin embargo 


\section{Tabla 2}

Matriz de Componentes

\begin{tabular}{|c|c|c|c|c|c|c|c|}
\hline Item & Factor 1 & Factor 2 & Factor 3 & Item & Factor 1 & Factor 2 & Factor 3 \\
\hline $\mathrm{R} 1$ & & &, 351 & $\mathrm{R} 44$ &, 090 & & \\
\hline R3 &, 355 & & & R45 & & , 468 & \\
\hline R4 & & ,229 & & R46 & ,395 & & \\
\hline R5 & & ,228 & & $\mathrm{R} 47$ & & ,214 & \\
\hline $\mathrm{R} 10$ & ,589 & & & $\mathrm{R} 48$ & & & ,044 \\
\hline R11 & & ,342 & & R49 & & , 494 & \\
\hline R12 & ,502 & & & R50 & & ,224 & \\
\hline R13 & ,409 & & & R51 & & &, 585 \\
\hline R14 & ,428 & & & R52 & & & ,396 \\
\hline R15 & & ,343 & & R54 & & &, 820 \\
\hline R16 & & &, 515 & R55 & & & ,447 \\
\hline R17 & & ,590 & & R56 & & ,567 & \\
\hline R18 & & &, 527 & R57 & & & ,296 \\
\hline R19 & & , 080 & & R58 & , 490 & & \\
\hline $\mathrm{R} 20$ & & & , 425 & R60 & ,329 & & \\
\hline R24 & ,457 & & & R61 & &, 533 & \\
\hline $\mathrm{R} 25$ & ,548 & & & R62 & ,277 & & \\
\hline R26 & & ,301 & & R63 & & ,369 & \\
\hline $\mathrm{R} 27$ & & ,381 & & R64 & & & ,565 \\
\hline R28 & & & , 159 & R65 & & , 470 & \\
\hline R30 & ,289 & & & R66 & ,337 & & \\
\hline R31 & & ,349 & & R68 & & ,306 & \\
\hline R33 & & & ,417 & R69 & ,412 & & \\
\hline R34 & & ,200 & & $\mathrm{R} 70$ & ,645 & & \\
\hline R35 &, 358 & & & R71 & ,606 & & \\
\hline R37 & & &, 421 & R72 & ,708 & & \\
\hline R38 & & & ,536 & $\mathrm{R} 73$ & ,409 & & \\
\hline R39 & & &, 517 & R74 & & ,276 & \\
\hline $\mathrm{R} 40$ &, 530 & & & R75 & ,634 & & \\
\hline R42 & ,441 & & & R76 & &, 376 & \\
\hline $\mathrm{R} 43$ & & ,644 & & & & & \\
\hline
\end{tabular}


hacen referencia a independencia (5) y a motivaciones (34 y 50), características relevantes para esta dimensión teórica.

El Factor 3 se corresponde con la dimensión Características Socioafectiva y hace alusión a aquellos ítems que miden motivación, independencia, responsabilidad y compromiso, niveles de energía, entre otras. Este factor explica el 8,546\% de la varianza saturando en él los ítems $1,16,18,20,33,37,38,39,51,52,55,57$ y 64 (ver matriz de componentes en el anexo). Pese a que los ítems 28 y 54 presentan también un peso factorial elevado en el componente 1 y el ítem 48 en el componente 3, se decidió dejarlos en la dimensión tres, puesto que teóricamente hacen alusión a niveles de energía (28 y 54) y sentido de justicia (48) características constituyentes de la subescala Socioafectiva.

\section{Indicadores de Validez}

Para validar esta escala se ha procedido de modo de realizar una serie de contrastes de medias (t Student) entre los sujetos "Talentosos Académicamente" y la "Población General" para cada uno de los niveles educativos, en cuanto a las medidas referidas a las características centrales de los estudiantes poseedores de Talento Académico (percepción de logros académicos en las asignaturas de mayor interés, reconocimiento social acerca de la creatividad de sus trabajos realizados, nivel de expresión de emociones y reconocimiento social de sus propias habilidades).

El primer paso consistió en segmentar la muestra de modo de generar puntajes de corte, tanto para quinto básico $(\mathrm{N}=127)$ como para primero medio $(\mathrm{N}=141)$, que permitiesen dividir la muestra en dos grupos: talentosos académicamente y población general. Este corte se realizó sumando una desviación típica a la media, de modo de asegurar (bajo el supuesto de normalidad en la distribución de las características de talento académico) que un porcentaje cercano al $15 \%$ quedara en el rango superior, tal y como lo predicen los teóricos vinculados a este ámbito de estudios (Gagné, 2000; Renzulli, 2004).

Para quinto básico la media fue de 4,84 y la desviación estándar ,54, siendo el punto de corte 5,38. El grupo "Talentosos Académicamente" quedó conformado por el 15,7\% $(\mathrm{N}=20)$ de los estudiantes y la "Población General" por el 84,3\% $(\mathrm{N}=107)$. En el caso de primero medio la media fue de 4,6 y la desviación estándar ,54, teniendo un punto de corte de 5,14. De este modo el grupo de "Talentosos Académicamente" quedó constituido por $16,3 \%(\mathrm{~N}=23)$ y la "Población General" por el 83,7\% $(\mathrm{N}=118)$ de estudiantes (ver tabla de frecuencia en el anexo).

Una de las hipótesis establecidas en este estudio es que se encontrará una correlación positiva entre la escala Edita y el Cuestionario del Alumno. Para contrastar dicha hipótesis se realizó el análisis de correlación (Pearson), obteniéndose una correlación positiva, alta y significativa $(\mathrm{r}=, 81 ; \mathrm{p}<0,01)$, lo que estaría indicando que ambas escalas están orientadas a medir el constructo Talento Académico. Este dato constituye un indicador de validez convergente para la escala diseñada.

Otra de las hipótesis es que se esperaba encontrar diferencias significativas entre los “Talentosos Académicamente" y la "Población General”, tanto para quinto básico como para primero medio, en relación a la percepción de logros académicos en las asignaturas científico-humanistas de su interés. Para comprobar dicha hipótesis se realizaron contrastes de media (t Student) para ambos niveles educativos. En el caso de quinto básico se encontraron dichas diferencias entre las medias de ambos grupos $(\mathrm{t}(118)=5,42 ; \mathrm{p}<0,05)$ 
en la percepción de logros académicos en la asignatura de mayor interés. Para primero medio dichas diferencias significativas entre las medias de ambos grupos $(\mathrm{t}(139)=4,05$; $\mathrm{p}<0,05)$ también aparecieron. Es decir, independiente del nivel educativo, el grupo que presenta mayores niveles de características de Talento Académico percibe significativamente mejores resultados académicos en las asignaturas de su interés que la población general. Además se encontraron correlaciones significativas entre los puntajes de la escala y la percepción de logros académicos en el área de su interés tanto para quinto básico $(\mathrm{r}=, 36 ; \mathrm{p}<0,01)$ como para primero medio $(\mathrm{r}=, 3 ; \mathrm{p}<0,01)$.

Además se realizó un análisis de correlación de Pearson entre la media total de la escala y el promedio de notas obtenido en el área académica Científico-Humanista de su interés, tanto para quinto como para primero medio. Los resultados obtenidos indican una correlación significativa tanto para quinto básico $(\mathrm{r}=, 361 ; \mathrm{p}<0,01)$ como para primero medio $(r=, 36 ; p<0,01)$. Es decir, pese a que ambas correlaciones son moderadas, su dirección positiva y su significación estarían indicando que los estudiantes que tienen mayores niveles de características de Talento Académico presentan un promedio de notas más alto en las asignaturas de su interés, independiente del nivel educativo al que pertenezcan.

Frente a otra de las hipótesis establecidas en el estudio en que se esperaban diferencias significativas entre los "Talentosos Académicamente" y la "Población General", tanto para quinto básico como para primero medio en relación a la percepción sobre el reconocimiento social de la calidad creativa de sus trabajos, se realizaron contrastes de media (t Student) para ambos niveles educativos. Se encontraron diferencias significativas tanto para quinto básico $(\mathrm{t}(81)=6,83 ; \mathrm{p}<0,05)$ como para primero medio $(\mathrm{t}(137)=3,63 ; \mathrm{p}<0,05)$. Por lo tanto se puede decir que el grupo que presenta mayores niveles de características de Talento Académico percibe un mayor reconocimiento social sobre la creatividad de sus trabajos que la población general, independiente del nivel educativo al que pertenezca. Asimismo se encontraron correlaciones significativas entre la escala y la percepción sobre el reconocimiento social de la calidad creativa de los trabajos que realizan, ya sea para quinto básico $(\mathrm{r}=, 49 ; \mathrm{p}<0,01)$ como para primero medio $(\mathrm{r}=, 52 ; \mathrm{p}<0,01)$.

Por otra parte, se hallaron diferencias significativas $(\mathrm{t}(138)=2,44 ; \mathrm{p}<0,05)$ entre los "Talentosos Académicamente" y la "Población General" de primero medio, en su percepción acerca del reconocimiento social de sus habilidades. Por lo tanto se podría afirma que el grupo que presenta mayores niveles de características de Talento Académico tiene una mejor percepción del reconocimiento social de las habilidades que poseen en comparación a la población general. Estas diferencias no aparecieron en quinto básico $(t(125)=1,93 ; p>0,05)$, aunque el resultado obtenido tiene una significancia levemente superior a 0.05 . En todo caso, se encontró una correlación significativa entre la escala Edita y la percepción sobre el reconocimiento social de las habilidades que poseen los participantes, tanto para quinto básico $(\mathrm{r}=, 24 ; \mathrm{p}<0,01)$ como para primero medio $(\mathrm{r}=, 34 ; \mathrm{p}<0,01)$.

Frente a la hipótesis que apuntaba a detectar la diferencia entre los grupos "Talentosos Académicamente" y la "Población General" en relación al grado de expresión verbal de las emociones, no se encontraron diferencias significativas ni para quinto básico $(\mathrm{t}(125)=1,02 ; \mathrm{p}>0,05)$ ni para primero medio $(\mathrm{t}(139)=0,104 ; \mathrm{p}>0,05)$. Es decir, todos los estudiantes, presenten o no características asociadas al Talento Académico, verbalizan sus emociones en grado similar. Se encontraron además correlaciones significativas entre 
la escala y la expresión de las emociones en quinto básico $(\mathrm{r}=, 19 ; \mathrm{p}<0,05)$, no así para primero medio $(\mathrm{r}=-, 03 ; \mathrm{p}>0,05)$.

Respecto de la hipótesis que busca detectar diferencias entre el grupo "Talentosos Académicamente" y la "Población General" en la percepción del involucramiento familiar en el proceso educativo, se encontraron diferencias significativas para el grupo de primero medio $(\mathrm{t}(33)=2,12 ; \mathrm{p}<0,05)$, sin embargo para los grupos formados en quinto básico las diferencias no fueron significativas $(\mathrm{t}(124)=-0,77 ; \mathrm{p}>0,05)$. Esto estaría indicando que los estudiantes de primero medio que poseen mayores características de Talento Académico perciben un mayor involucramiento familiar que la población general. Por otro lado, los estudiantes de quinto básico independiente de los niveles de características de Talento Académico que posean, no perciben significativamente distintos los niveles de involucramiento familiar en su proceso educativo. Además en el análisis de correlaciones ésta fue significativa para primero medio $(\mathrm{r}=, 2 ; \mathrm{p}<0,05)$ no así para quinto básico $(\mathrm{r}=, 01 ; \mathrm{p}>0,05)$.

Una de las hipótesis más relevantes trabajadas en el estudio se relaciona con el hecho de que no se encontrarían diferencias en los niveles de manifestación de características de Talento Académico en los diferentes niveles socioeconómicos. Tras el contraste, se concluye que no existen diferencias significativas tanto en quinto básico $(\mathrm{F}(2,124)=1,27$; $\mathrm{p}>0,05)$ como en primero medio $(\mathrm{F}(2,138)=1,35 ; \mathrm{p}>0,05)$. Dado lo anterior, se puede afirmar que las manifestaciones de Talento Académico no dependen del nivel socioeconómico de la muestra.

Finalmente, se verificó si no existen diferencias por sexo en la expresión de características de Talento Académico. Para esto se realizó la prueba t Student para cada uno de los niveles educativos, tras la cual se concluye que no existen diferencias significativas ni en primero medio $(\mathrm{t}(139)=-1,32 ; \mathrm{p}>0,05)$ ni en quinto básico $(\mathrm{t}(125)=2,077 ; \mathrm{p}>0,05)$ en la manifestación de características de talento académico entre hombres y mujeres. Es decir, la posesión de Talento Académico no depende del sexo de los participantes.

\section{DISCUSION}

Los resultados obtenidos en el proceso de construcción y validación de la Escala de Identificación de Talento Académico, Edita, son satisfactorios. El instrumento demuestra ser una herramienta fiable para medir las características asociadas al Talento Académico. Así lo indican los coeficientes de fiabilidad obtenidos en la muestra para la prueba total y para cada una de las subescalas que la componen.

Por otra parte se han encontrado correlaciones significativas y positivas entre las subescalas, las que al no ser tan elevadas podrán estar indicando que miden distintos aspectos del constructo Talento Académico.

La estructura factorial obtenida es concordante con las dimensiones teóricas propuestas previa construcción de los ítems (Conocimiento y Habilidades de Aprendizaje, Creatividad y Productividad y Características Socioafectivas). Pese a que existen algunos ítems con carga factorial compartida en más de un factor, estos pueden ser forzados a la dimensión teórica concordante, no comprometiendo el valor psicométrico de la prueba. 
Además, se pudo comprobar la validez convergente encontrándose una alta correlación entre esta escala y el Cuestionario para el Alumno, prueba que actualmente se utiliza en Chile para medir el constructo Talento Académico. Esta correlación al ser significativa y positiva estaría indicando que la escala Edita logra medir satisfactoriamente dicho constructo.

Las pruebas estadísticas realizadas no señalan diferencias entre los dos niveles educativos que conformaban la muestra (quinto año básico y primer año medio), lo que implica que esta escala mide satisfactoriamente el constructo Talento Académico en ambos grupos.

Dentro de las predicciones relevantes que se consideraron como indicadores de validez, se encontró que independiente del nivel educativo al que pertenezcan los estudiantes, los que presentan Talento Académico perciben mejores resultados académicos en las asignaturas de su interés y, además, efectivamente tienen un mejor promedio de notas en dichas asignaturas en comparación a la población general. Lo que confirma la teoría que señala que los estudiantes que poseen Talento Académico presentan mayores logros académicos en las asignaturas científico-humanistas de su interés.

Respecto a otras de las predicciones relevantes como indicadores de validez, a través de este estudio es posible afirmar efectivamente que el grupo que presenta mayores niveles de Talento Académico, tanto en quinto básico como en primero medio, percibe un mayor reconocimiento social sobre las habilidades que posee y sobre la calidad creativa de sus trabajos, independiente del nivel educativo al que pertenezcan.

Desde la teoría los estudiantes que poseen Talento Académico presentan un discurso muy intelectualizado. Sin embargo, desde este estudio es posible afirmar que los estudiantes "Talentosos Académicamente" en ambos niveles educativos no expresan de manera diferente sus emociones que los que conforman la "Población General". Esto puede explicarse debido a que, en términos generales, el ítem fue poco preciso al momento de indagar sobre el modo en que ellos expresan sus emociones.

La predicción respecto a la percepción del involucramiento familiar de los estudiantes "Talentosos Académicamente" arrojó diferentes resultados para los dos niveles educativos. Dichos participantes de primero medio efectivamente perciben un mayor involucramiento familiar en su proceso educativo en relación a la "Población General", no evidenciándose estas diferencias en los estudiantes de quinto básico. Estas discrepancias en los resultados pudieron deberse a que los participantes de quinto año valoraron el ítem como el involucramiento familiar desde la participación en las actividades que involucra la educación formal y no desde la estimulación o motivación que sus familias les transmiten hacia el aprendizaje.

Otra de las predicciones que se realizaron en este estudio, la independencia de las manifestaciones de Talento Académico tanto del sexo como del nivel socioeconómico de los estudiantes para ambos niveles educativos era de suma importancia, comprobándose que las manifestaciones de Talento Académico se distribuyen de forma equivalente entre hombres y mujeres, así como también en los diferentes niveles socioeconómicos. Esto permitiría afirmar que el Talento Académico es una característica que se distribuye en forma homogénea en la población, pero como lamentablemente se le vincula de manera errada a éxito académico, se ha generado en lo social una imagen que discrimina a sectores desfavorecidos. Sectores en los cuales ha quedado comprobado por medio de este estudio, poseen los mismos niveles de Talento Académico que los estratos socioeconómicos medios 
y altos, sólo que no poseen las mismas oportunidades ni condiciones para desarrollarlo y potenciarlo. De aquí que sería necesario realizar estudios sobre cómo el talento, que se supone una habilidad adquirida y entrenada, puede haberse desarrollado en los estudiantes de todos los niveles económicos y de diferentes realidades educativas.

Además, la distribución del porcentaje de estudiantes que poseen características de Talento Académico encontrada a través de este estudio (16,3\%) se condice con la planteada por Renzulli, quien señala que la población poseedora de Talento Académico alcanzaría hasta un $20 \%$, y no el 2,5\% como lo proponen antiguas teorías. Esta situación estaría reafirmando que existe un alto porcentaje de Talento Académico en la población, porcentaje que no ve satisfechas sus necesidades educativas especiales, por la ausencia de políticas sociales que permitan su integración efectiva, situación que se denota con mayor fuerza en los niveles socioeconómicos más deprivados.

La importancia de los resultados obtenidos en este estudio radica en el hecho de que se ha podido comprobar que al momento de considerar a un estudiante como Talentoso Académicamente, es fundamental tomar en cuenta las características asociadas a este constructo como complemento a los niveles de inteligencia que éste posea, siendo la capacidad intelectual una condición necesaria pero no suficiente del Talento Académico. Por lo tanto, los resultados obtenidos se condicen con la teoría que afirma que el constructo Talento Académico es más amplio que el de Inteligencia, puesto que se distribuye de forma más homogénea que este último en la población. De este modo, dada la alta confiabilidad de la escala es posible afirmar que el instrumento construido está midiendo características asociadas al Talento Académico y no a la Inteligencia. De este modo, en el caso de que un estudiante que posea niveles promedio de inteligencia obtenga en esta escala altos niveles de características asociadas al Talento Académico, será posible promover recomendaciones y prácticas pedagógicas orientadas a satisfacer sus necesidades educativas, y no sólo para seleccionar estudiantes para formar parte de programas destinados a Talentos Académicos.

Desde la perspectiva de las mejorías posibles a esta escala, es necesario considerar para estudios posteriores la principal limitación que presenta: su falta de grupos educativos de niveles intermedios, ya que por una decisión práctica sólo se consideró quinto básico y primero medio (que son los niveles con los que se trabaja en el programa DELTA de la Universidad Católica del Norte, y debido a que esta escala fue pensada originalmente para su utilización en dicho contexto).

En conclusión la escala parece funcionar bien, en cuanto logra diferenciar de modo satisfactorio aquellos niños, niñas y jóvenes que manifiestan características de Talento Académico de aquellos que no las manifiestan, demostrando ser válida y confiable para estudiantes de quinto básico y primero medio de establecimientos municipalizados, subvencionados y particulares de este entorno social.

\section{REFERENCIAS}

Arancibia, V., Herrera, P. \& Strasser, K. (2004). Manual de Psicología Educacional. Santiago: Ediciones Universidad Católica de Chile.

Benito, Y. (1998). Trastornos emocionales y problemática de adaptación en alumnos superdotados. Ponencia presentada en el Primer Congreso de Educación de Alta Inteligencia, Mendoza, Argentina. 
Benito, Y. \& Alonso, J. (2004). Sobredorados, Talentosos, Creativos y Desarrollo Emocional. Loja: UTPL.

Bralic, S. \& Romagnoli, C. (2000). Niños y Jóvenes con Talento: Una Educación de Calidad para Todos. Santiago: Dolmen Ediciones S.A.

Chile, Comisión de Expertos de Educación Especial. (2004). Nueva Perspectiva y Visión de la Educación Especial. Santiago: La Nación.

Colombia, Instituto Alberto Merani (2005). IAM. Extraído el 10 de enero, 2006, de http://www. institutomerani.edu.co.

Cronbach, L. (1998). Fundamentos de los Test Psicológicos. Madrid: Biblioteca Nueva.

Flanagan, A. \& Arancibia, V. (2005). Talento académico: un análisis de la identificación de alumnos talentosos efectuada por profesores. Psykhe, 14 (1), 121-135.

Gagné, F. (2000). Un modelo diferenciador de dotación y talento. Disponible en: http://www.puc. cl/pentauc.

García, F. \& Doménech, F. (2005). Motivación, aprendizaje y Rendimiento Escolar. Reme, 0(1). Extraído el 27 de julio, 2005 de http://www.reme.uji.es.

García, M. \& González, J. (2004). Fundamentos de la Educación para la Excepcionalidad. Bogotá: Javegraf.

Gardner, H. (1994). Estructuras de la Mente: La Teoría de las Inteligencias Múltiples. México: Fondo de la Cultura Económica.

George, D. (1992). The challenge of the able child. London: David Fulton.

Goleman, D. (1996). La Inteligencia Emocional. Buenos Aires: Javier Vergara Editor S.A.

Landau, E. (1999). Talento Creatividad y Desarrollo Socioemocional. En S. Bralic \& C. Romagnoli (Eds.), Seminario Internacional: La Educación de los Alumnos con Talentos Académicos Destacados (pp. 159-178). Santiago: Dolmen Ediciones S.A.

Renzulli, J. (2004, noviembre). Cómo se Produce la Superdotación y Cómo Podemos Desarrollar Dotes y Talentos en la Gente Joven. Ponencia presentada en el Quinto Congreso Iberoamericano de Superdotación y Talento, Ciudad de Loja, Ecuador.

Renzulli, J. (2006). Entrevista en Radio Televisión Española. 15 de junio, 2006. http://www.rtve.es Rimm, S. (1994). Keys to parenting the gifted child. New Cork: Barron's Educational Series, Inc.

Sternberg, J. \& O’Hara, L. (2005, junio 3). Creatividad e Inteligencia. Cuadernos de Información y Comunicación, 10, Artículo 6. Extraído el 21 de julio, 2006 de http://www.ucm.es

Sternberg, R. (1985). Beyond I. Q. A triarchic theory of human intelligence. Cambridge: Cambridge University Press.

Terman, L. (1965). Inteligencia, El Interés y la Actitud. Buenos Aires: Paidós.

Terrassier, J. C. (1998). El síndrome de asincronía. Ponencia presentada en el Primer Congreso de Educación de Alta Inteligencia, Mendoza, Argentina.

Weschler, D. (1998). Test de Inteligencia para Niños WISC-III Manual. Buenos Aires: Paidós. 
